\title{
12-Week Resistance Training with Breast Cancer Patients during Chemotherapy: Effects on Cognitive Abilities
}

\author{
Freerk T. Baumann $^{\mathrm{a}} \quad$ Nina Drosselmeyer ${ }^{\mathrm{a}} \quad$ Aleksandra Leskaroski $^{\mathrm{b}}$ \\ Axel Knicker $^{\mathrm{a}}$ Holger Krakowski-Roosen $^{\mathrm{c}}$ Eva M. Zopf ${ }^{\mathrm{a}}$ Wilhelm Bloch ${ }^{\mathrm{b}}$ \\ aDepartment of Molecular and Cellular Sports Medicine, Institute for Cardiovascular Research and Sports Medicine, \\ German Sports University Cologne, \\ bInterdisciplinary Breast Center/University Hospital of Cologne, \\ ${ }^{\circ}$ German Cancer Research Center Heidelberg, Germany
}

\author{
Keywords \\ Breast cancer - Resistance training · Cancer - Sports . \\ Cognition
}

\section{Introduction}

Rates of cognitive impairment as high as $75 \%$ have been reported by breast cancer $(\mathrm{BCa})$ patients during and after chemotherapy, particularly concerning attention and memory $[1,2]$. The pathophysiology and incidence of this phenomenon, also referred to as 'chemobrain', is mostly unclear. However, the recent literature indicates that several factors are responsible for the cognitive changes, including physiological parameters and psychological stress [1, 3].

Numerous studies confirm the positive effects of physical activity on cognitive function. Not only a reduced risk of suffering from dementia has been observed but, first and foremost, improvements in concentration, attention control (top-down control), and stimulus processing [4, 5]. To date, the influence of resistance training on cognitive functioning in patients receiving chemotherapy has not been studied. The purpose of this prospective non-randomized controlled trial was to examine the effects of a 12-week resistance training on the cognitive abilities of BCa patients during chemotherapy.

\section{Methods}

Seventeen BCa patients (tumor stages I-III) receiving (neo-)adjuvant chemotherapy were recruited from the Breast Center of the University Hospital of Cologne, Germany. The protocol was approved by the ethics committee of the German Sports University, Cologne, and all patients provided written informed consent prior to participation. 9 patients took part in the intervention group (IG) while 8 preferred the control group
(CG). Patients in the IG participated in a 60 -min session of resistance training twice a week for 12 weeks, performing 3 sets of 8-12 repetitions of 10 different exercises at $55-75 \%$ of their hypothetical 1-repetition maximum. Exercise sessions were supervised and performed at a healthorientated fitness studio.

Cognitive functions were assessed by 4 neuropsychological tests. The MEMO memory test [6] was applied to test verbal memory, the Wilde intelligence subtest (WIT) [7] to determine the working memory, and the $\mathrm{d} 2$ test of attention, consisting of 2 different tests, to provide information about focused attention and concentration abilities [8]. Assessments were conducted at the beginning (pre) and at the end (post) of the 12-week physical intervention in the IG, whereas the CG was only tested at the end of the trial. The pre-test was conducted during chemotherapy and the post-test 1-2 weeks after its termination.

Statistical analyses consisted of a paired/dependent t-test (type 1) to assess intergroup changes in the IG and an independent t-test (type 2, 2 samples, same variance) to identify differences between the IG and the $\mathrm{CG}$ at the end of the trial.

\section{Results}

The mean age of the IG members was $46.6 \pm 6.9$ years and that of the CG members was $52 \pm 5.5$ years (IG vs. CG, $\mathrm{p}=$ non-significant (n.s.)).

In the $\mathrm{d} 2$ test of attention, the scores significantly improved within the IG after exercising for 12 weeks (IG-pre vs. IGpost, $\mathrm{p}=0.049)$, whereas no significant differences were observed in comparison with the CG (CG-post vs. IG-pre or IG-post, $p=$ n.s.). The error rate of the $\mathrm{d} 2$ test decreased significantly in the IG by 1.12 points between the pre- and post-test (IG-pre vs. IG-post, $\mathrm{p}=0.017$ ). It was also significantly lower in the IG when the pre- and post-test of the IG were compared to the post-test of the CG (IG-pre vs. CG-post, $\mathrm{p}=0.040$; IG-post vs. CG-post, $\mathrm{p}=0.019$ ).

Short-term verbal memory improved only marginally in the IG at the end of the training period (IG-pre vs. IG-post,

\section{KARGER}

Fax +497614520714

Information@Karger.de

www.karger.com (c) 2011 S. Karger GmbH, Freiburg

$1661-3791 / 11 / 0062-0142 \$ 38.00 / 0$

Accessible online at:

www.karger.com/brc
Dr. Freerk T. Baumann

Deutsche Sporthochschule Köln

Institut für Rehabilitation und Behindertensport

Am Sportpark Müngersdorf 6, 50933 Köln, Germany

Tel. +49 221 4982-4821, Fax -8370

f.baumann@dshs-koeln.de 
$\mathrm{p}=0.163)$. However, the post-test results of the IG where significantly better, by 1-3 points compared to those of the CG (IG-post vs. CG-post, $\mathrm{p}=0.048$ ).

In the WIT, patients in the IG scored significantly better after the exercise intervention (IG-pre vs. IG-post, $p=0.049$ ). No significant differences were observed between the IG and the CG.

\section{Discussion}

The described trial examined the correlation between 'physical activity (resistance training)' and 'cognitive function' in BCa patients undergoing chemotherapy. Significant cognitive improvements in BCa patients after participating in a 12-week resistance training where measured in 3 out of 4 tests. The post-test scores of the CG were lower in all 4 tests when compared to those of the IG. To our knowledge no comparable results have been reported so far. This study therefore provides preliminary evidence that resistance training might have a positive effect on cognitive functions in $\mathrm{BCa}$ patients receiving chemotherapy. The exact mechanisms of this effect are still unclear; however, hormonal, cerebral, and psychological aspects may play a role. Limitations to this study include the small sample size $(n=17)$, lack of randomization, missing baseline assessments for the CG, and lack of a comprehensive intelligence test and educational/occupational diagnostics for both groups prior to chemotherapy/intervention. Furthermore, the cognitive tests were not administered by a psychologist and a learning effect cannot be excluded. Further studies are necessary to provide further experimental support for these results and to overcome the admitted shortcomings of this study. Particularly the BCa patients' hormone status and chemotherapeutic regimen should be considered in future investigations.

\section{Disclosure Statement}

The authors declare no conflict of interest.

\section{References}

1 Myers JS: Chemotherapy-related cognitive impairment. Clin J Oncol Nurs 2009;13:413-421.

2 Stewart A, Bielajew C, Collins B, Parkinson M, Tomiak E: A meta-analysis of the neuropsychological effects of adjuvant chemotherapy treatment in women treated for breast cancer. Clin Neuropsychol 2006;20:76-89.
Ahles TA, Saykin A.J: Candidate mechanisms for chemotherapy-induced cognitive changes. Cancer 2007;7:192-201.

4 Larson EB, Wang L, Bowen JD, McCormick WC, Teri L, Crane P, Kukull W: Exercise is associated with reduced risk for incident dementia among persons 65 years of age or older. Ann Intern Med 2006;144:73-81.
5 Hillman CH, Erickson KI, Kramer AF: Be smart, exercise your heart: exercise effects on brain and cognition. Nat Rev Neurosci 2008;9:58-65.

6 Schaaf A, Kessler J, Grond M, Fink GR: MemoTest. Goettingen, Hogrefe, 1994.

7 Jäger AO, Althoff K: Der WILDE-Intelligenz-Test (WIT). Handanweisung. Göttingen, Hogrefe, 1983.

8 Brickenkamp R: Aufmerksamkeits-BelastungsTest (d2), ed 8. Göttingen, Hogrefe, 1994. 